\title{
Physical activity
}

\section{Rapporteur's report}

Chair: Michael Sjöström ${ }^{1}$

Rapporteur: Ken Fox ${ }^{\star, 2}$

${ }^{1}$ Karolinska Insitutet, Stockholm, and Örebro University, Sweden

2 Department of Exercise and Health Sciences, University of Bristol, UK

*Correspondence: Email K.R.Fox@bristol.ac.uk

Keywords

physical activity, exercise, health, obesity, health promotion

\section{Presentations}

Health benefits of physical activity

Lars Bo Andersen, University of Copenhagen, Denmark

Consumer attitudes to physical activity, body weight and health among EU populations

John Keamey, Institute of European Food Studies, Trinity College, Dublin

Recommendations for primary health care to promote healthy physical activity

Seppo Miilunpalo, UKK Institute, Tampere, Finland

\section{The case for physical activity}

There was consensus that physical activity is vital for health. There is general support for physical activity by academics and the general public because there is an intuitive belief that physical activity is good and a natural aspect of healthy living that improves physical and mental health. This is evidenced by growth in the promotion of physical activity from grass roots in many member states, such as exercise by prescription schemes in the UK. These have developed through the enthusiasm of exercise professionals and the enjoyment of participants, largely in the absence of hard evidence of effectiveness or top down policy.

The research base for physical activity and its impact on health has developed largely in the past 15 years and so is relatively new. It also has not received high levels of funding. However, the evidence that physical activity helps prevent coronary heart disease, colon cancer, obesity, and type 2 diabetes is now strong and well accepted by leading health and medical authorities.

The case for physical activity has primarily been made on its impact on cardio-respiratory health but convincing evidence is emerging for a powerful effect on metabolic fitness (blood fats, insulin sensitivity and glucose tolerance) largely through muscle and substrate utilisation change. This may explain the evidence that fatness appears much less harmful when accompanied by regular physical activity and improved fitness. In addition, stronger evidence is now emerging on the effects of physical activity on mental health. There is little evidence as yet of the cost-effectiveness of physical activity promotion schemes or campaigns.

\section{A natural activity-nutrition partnership}

There are many areas where diet, nutrition and physical activity overlap. These were explored in depth through the Institute of Life Sciences (Europe) Workshop on Diet and Physical Activity Interactions for Health ${ }^{1}$.

Activity and nutrition scientists need to come together on topics such as appetite and activity interactions, exercise and food interactions on substrate utilisation, the effects of food and activity on well-being, the problem of dietary restraint and inactivity in adolescent girls, and joint approaches to measurement.

Obesity treatment and prevention offers the critical ground for partnership. Obesity is providing the leading health challenge of the new millennium and most European states are already seeing dramatic increases in incidence. Obesity is closely associated with coronary heart disease and the rising incidence of diabetes, as well as several other problems such as cancer, osteo-arthritis, and respiratory problems. In addition to health, there are serious implications for safety policy and legislation with larger people requiring different specifications for seating and loadbearing equipment. 
Table 1 Obesity: A poor adaptation to modern living

\begin{tabular}{ll}
\hline Eating & Activity \\
\hline Energy dense food is readily available for 24 & Reduced availability due to cars and labour-saving devices \\
hours a day & Drive to exercise is weak in the absence of a stressor \\
Drive to eat (hunger) is strong & Drive to stop exercise is strong (fatigue, discomfort) \\
Drive to stop eating (satiation) is weak & Exercise is often not seen as pleasurable (particularly by \\
Eating is pleasurable and sociable & those who do little) \\
No viable substitute for eating and drinking & Sitting down (TV) provides a pleasurable alternative
\end{tabular}

Within the study of obesity, energy intake and expenditure require simultaneous assessment. The problem arises from an inadequate adaptation to accessibility to food and labour-saving technology (see Table 1). The interactive effects of diet and activity on metabolic health may also provide the key to understanding Syndrome $\mathrm{X}$ (or the metabolic syndrome).

With regard to the delivery of treatment, changing dietary habits and activity behaviour for public health both face similar challenges. The delivery of support services will be inevitably tied together, and professional training will have considerable overlap.

It is clear that the problem of obesity will not be adequately tackled without a joint approach and the recommendations from Eurodiet to the Commission require a strong emphasis on an activity-nutrition partnership.

In addition, physical inactivity is a relatively new problem and as such the underpinning sciences and academic and professional organisations are new and underfunded. The problem of inactivity is often seen as everyone's and no-one's responsibility as its solutions lie with policy in transport, health, education and media. A firm partnership with nutrition could act as a catalyst to placing health enhancing physical activity higher on the list of priorities.

\section{Recommendations}

General. A strong statement is required early in the documentation declaring support for physical activity policy and the need to develop a partnership. This is particularly required where the prevention and treatment of obesity as a major challenge is addressed.

Working Party 1. There is potential to further update and strengthen the case for activity, especially in the light of the recommendation for a focus on obesity. Outcomes of a recent American College of Sports Medicine Round Table on the prevention and treatment of obesity and its comorbidities were published in November 19992. Lars Bo Anderson showed that there is scope to extend the case for activity in reduction in colon cancer, effects in older people, and on mental health.

The recommendation for activity is a PAL value of 1.75. This energy expenditure value has an evidence base and is appropriate where the focus is on obesity. It would be helpful to also state the value in $\mathrm{kcals} / \mathrm{kg} /$ day or week. Motion sensors (accelerometers) are able to produce such figures and this is likely to replace self-report even in large sample studies in the next two or three years as technology advances and price of instruments drops.

Working Party 2. Consider the influence of levels of activity on FBDGs, particularly the impact of patterns of sedentariness (TV watching etc) versus high levels of activity.

Working Party 3. There is strong support for the three stage model of targeting, settings, and approaches adopted for addressing promotion of healthier eating and physical activity.

1. Targeting and tailoring to populations.

John Kearney showed clear evidence of large national differences in levels of activity but also perceptions of the benefits and importance of activity for health. There is a considerable latitude gradient with some Mediterranean countries such as Greece and Portugal indicating culture-specific attitudes that activity is neither as beneficial or important as in Scandinavian countries. This is associated with less activity and higher incidence of obesity. Clearly this points towards public health campaigns tailored to national idiosyncrasies. Similarly, within nations there is a clear socio-economic gradient for activity participation. Sub-cultural differences in value systems regarding activity must be addressed if 
campaigns are to be effective. There will also be important gender and age differences.

2. Approaches and settings.

Seppo Miilunpalo made the case that different approaches will be required with different populations and sub-populations. Persuasion and education are still seen as important as shown by the national differences in attitudes so media campaigns are critical to setting the agenda and language for health promotion. More targeted approaches are required in a range of settings. Schools are seen as critical as they have a captive audience across all social groups and can be influential on attitudes, values, self-perceptions and behaviour that track through to adulthood. Similarly, the workplace and primary care offer important settings for activity promotion. It is absolutely critical that approaches are aimed at environmental change as well as individual change. Activity friendly localities are required and this will involve town planning, transport and architecture policy. Some degree of coercion may be necessary to encourage people to use cars less and walk and cycle more.

3. Professional accreditation.

There is a need to produce professionals with chartered or registered status who can address dietary and activity needs of individuals and groups. This would involve a range of skills and technical knowledge in counselling and exercise and dietary change. Masters programmes in Public Health Nutrition, and Exercise and Health Sciences offer an important preparation.

Working party 4. Documentation needs to address physical activity directly, not only as an element of lifestyle. It could emphasise the need for a comprehensive and coherent EU physical activity policy, general recommendations for physical activity promotion and the need for greater research funding in this area.

This would support existing pan-European organisations that have physical activity promotion agenda such as the European Heart Network, the European College of Sport Sciences, the European Network for Public Health Nutrition and the European Network for Health Enhancing Physical Activity (HEPA).

This in turn would help member states develop their own forum and lobby for physical activity policies, professional accreditation and science base.

\section{Summary}

Eurodiet is to be commended for its degree of consideration of physical activity

It could extend its support even further through the documentation and recommendations

A tremendous opportunity exists to forge exciting and essential links between the two areas of science and policy making

\section{References}

1. Sjöström M (Ed.). ILSI (Europe) workshop on diet and physical activity: Interactions for health. Public Health Nutrition, 1999; 2 (Supplement 1).

2. Blair SN, \& Bouchard C (Co-Chairs). Physical activity and obesity: American College of Sports Medicine Consensus Conference. Medicine and Science in Sports and Exercise, 1999; 31(11) Suppl. S497.

\section{Abstracts}

\section{Health benefits of physical activity}

\section{Lars Bo Andersen*}

Institute for Physical Education and Sports Sciences, University of Copenhagen, Norre Alle 51, DK-2200 Copenhagen N, Denmark

*Correspondence: Email Ibandersen@ifi.ku.dk

Keywords

Physical activity, prevention, metabolic disease, cancer, osteoporosis

Scientific evidence on the relationship between physical activity and a number of common diseases have accumulated during the last decades. Today, most scientists agree that a high physical activity level reduces the risk of a number of diseases related to metabolic disorders, osteoporosis and some cancers as well as improvements in functional ability at all ages. Among the metabolic disorders are coronary 
heart disease (CHD), diabetes II, hypertension, and hyperlipidemia. Further, beneficial associations have been found between physical activity and a number of conditions where consensus of a causal relationship is not yet established. Physical activity may improve psychological well-being, the immune system, sleep, and low back pain among others.

The mechanisms behind the observed preventive effects of physical activity and its interaction with nutritional factors varies depending on the disease. Some possible mechanisms in relation to metabolic diseases, colon cancer and osteoporosis.are reviewed here.

Insulin sensitivity is a key factor in the causal chain to metabolic diseases. Insulin secretion is directly affected by the content of glucose in the blood (coming from the diet), but the glucose transport (GLUT-4 receptors in the plasma membrane of skeletal muscle) is increased by exercise. The atherosclerotic process is enhanced by a high content of saturated fat in the diet, but lipoprotein lipase activity is highly modifiable by exercise.

Some nutritional factors increase the risk of colon cancer, but exercise decreases the time the carcinogenic agents are in contact with the mucosa of the intestine.

Many elderly people eat so little that they get insufficient essential nutrients for bone remodelling, and supplements of D-vitamin and calcium have been shown to decrease the risk of osteoporotic fractures. Exercise increases the energy intake and therefore the intake of vitamins and minerals. It also decreases the risk of falling by improving strength and balance, and the strain on the bones is necessary for the remodeling process.

In most prospective studies relating physical activity level to the incidence rate of one of the abovementioned diseases, a doubled risk has been found in the sedentary compared to the most physically active. Looking at $\mathrm{CHD}$, which is responsible for almost half of all early deaths, this is in the same order as the other major risk factors. Compared to the other CHD risk factors, the prevalence of physically inactive people at risk is very high. Therefore, physical activity has the largest potential for prevention, and it has been estimated that the population attributable risk is around one third ${ }^{1}$. This may seem high, but is in fact an underestimation, because the relative risk of being sedentary is underestimated. Physical activity is a health behaviour that changes over time in the individual. During 10 years of follow-up in a prospective study ${ }^{2}$, less than half of those classified as sedentary at baseline were still sedentary after 10 years (and were therefore misclassified) and the same was the case for those classified as physically active at baseline. This dilution may result in a $50 \%$ underestimation of the relative risk.

\section{References}

1. Haapanen-Niemi N, Vuori I, Pasanen M. Public health burden of coronary heart disease risk factors among middle-aged and elderly men. Prev Med 1999; 28: 343-348.

2. Copenhagen Center for Prospective Population Studies (in press).

\title{
Consumer attitudes to physical activity, body weight and health among EU populations
}

\author{
John M. Kearney*, Michael J. Gibney
}

Institute of European Food Studies, Trinity College, Dublin 2, Ireland

*Correspondence: Email iefs @iefs.ie

\author{
Keywords \\ physical activity, obesity, consumer attitudes, bodyweight
}

\begin{abstract}
Objectives: :In most developed countries, the rise in obesity in recent years has been paralleled by rising levels of physical inactivity. A limiting factor to the promotion of physical activity in relation to health. has been a knowledge of consumer attitudes and beliefs about physical activity and body-weight. The objectives of the pan-EU survey were to assess: attitudes and beliefs about physical activity, bodyweight and health, current levels of activity / inactivity (self-reported), and prevailing levels of over-weight and obesity.
\end{abstract}

Metbods. A cross-sectional study in which quotacontrolled, nationally representative samples of approximately 1,000 adults from each country completed a face-to-face interview-assisted questionnaire. The survey was conducted in the 15 member states of the European Union between march and April 1997.

Results. Physical activity was not perceived to be among the top three priorities having the greatest influence on overall health in the EU. Smoking, food and stress were perceived to be more important. 
Those selecting physical activity were less likely to select stress as a factor important for health. Wide interstate variation was seen for the selection of physical activity ranging from $9 \%$ in Italy to $44 \%$ in Finland.
Conclusions. Such wide interstate variation in attitudes and beliefs towards physical activity has implications for programmes being developed to promote physical activity across the EU.

\title{
Recommendations for primary health care to promote healthy physical activity
}

\author{
Seppo Miilunpalo*
}

UKK Institute for Health Promotion Research, P.O. Box 30, 33500 Tampere, Finland

*Correspondence: Email seppo.miilunpalo@uta.fi

\author{
Keywords \\ Physical activity, health promotion, patient, education, \\ counselling
}

Diet and physical activity are both important health related lifestyle factors, and it is relevant to consider the role of primary health care (PHC) in the promotion of healthy nutrition and physical activity, both among patients and in the whole population. However, an intemational expert group identified several barriers for the development of preventive and health promotional activities in $\mathrm{PHC}^{1}$. There is a lack of appropriate preventive guidelines for PHC, and inadequate dissemination of guidelines when they do exist; lack of skill and knowledge in the area of prevention, and lack of incentives for practising prevention. In a survey concerning the counselling practices in Finland the primary care providers reported, consistently with the expert statement, that the shortage of common guidelines is one of the major obstacles in developing patient health education and counselling. ${ }^{2}$

As a consequence of PHC working methods, individual counselling is usually considered as the most relevant method for the promotion of healthy lifestyles. $^{3}$ However, there are two noteworthy remarks for this. First, although individual counselling fits as a part for every care providers' daily work it does not preclude the development and use of complementary methods, such as group education, printed or audio-visual materials, computer programs as well as community activities. Second, in the development of counselling one should be aware of the role differences between the professional groups. The different professionals may have common clients and shared responsibilities in prevention, care and rehabilitation, but they also have different tasks and expert areas, as well as different work traditions.
Thus it is essential to develop collaboration and mutual understanding between the care providers.

Since the need for promotional activities varies between the clients one should develop and adopt adequate and feasible methods for the assessment and monitoring physical activity. In addition, the care providers should have adequate training about the principles and methods of individual counselling in order to understand the behaviour change process, to assess the client's readiness for change and to set goals for the counselling, to understand the determinants of behaviour and to support the client's change process ${ }^{4}$. In addition, despite the individual's readiness for change, there may be obstacles in the individual's life situation, or e.g. in the environment, which resist the client's ability to change his or her behaviour and comply with the care provider's advice. Therefore it is necessary to support the adoption of healthy lifestyles by developing the environmental possibilities, facilities and social conditions ${ }^{5}$.

One should not forget the structural characteristics of the health care organisation, which to a large extent determine the working conditions ${ }^{6}$. Therefore, in every $\mathrm{PHC}$ unit the management of the unit should consider, how the care organisation can support the individual care providers' co-work and the adoption of purposeful working methods. The following recommendations can be given. In each health care organisation the unit should (a) adopt or develop local guidelines and common procedures, (b) plan how to develop and maintain the professional skills of the staff and (c) build partnership with other actors in the field of health promotion? 


\section{References}

1. The Victoria Declaration on Hearth Health: declaration of the advisory board of the International Heart Health Conference, Victoria, Canada, 1992.

4. primary health care. Patient Education Counseling 1995;25:317-328.

5. Prochasca JO, Velicer WF. The transtheoretical model of health behaviour change. Am J Health Promotion 1997;12:38-48.

6. Green L, Kreuter MW. Health promotion planning. An educational and environmental
2. Laitakari J, Miilunpalo S, Vuori I. The process and methods of health counseling by primary health care personnel in Finland: a national survey. Patient Education and Counseling 1997;30:61-70.

3. Miilunpalo S, Laitakari J, Vuori I. Strengths and weaknesses in health counseling in Finnish approach. Palo Alto, CA. Mayfield Publishing Co., 1991.

7. Donabedian A. The Quality of Care. How Can It Be Assessed? JAMA 1988;260:1743-1748.

8. Promotion of health-enhancing physical activity at local level. Expert recommendations. Publications of Ministry of health and social affairs 2000:1. Helsinki 2000 (in Finnish). 